\title{
Population-based surveillance of services provided to counseling and prenatal clients in a multi-state region by state health departments: A proposal
}

After September 1999, the Great Plains Genetics Service Network (GPGSN) Database Committee will no longer carry out annual surveillance of genetic services offered to counseling and prenatal clients in the eight-state area of Arkansas, Iowa, Kansas, Missouri, Nebraska, North Dakota, Oklahoma, and South Dakota. The Database Committee reports for 19871997 summarized results on 160,643 counseling and prenatal clients. The 1997 Report included descriptive summary statistics on 8,790 counseling-evaluation clients and 8,915 women who received prenatal services. ' A summary of the 1998 dataset and a review paper summarizing 4 years of data for 1994-1997 should be available next year (personal communication, 1999). Thus far GPGSN data have been used to modify existing programs for counseling or prenatal clients, or to start new ones. GPGSN data were utilized to document the need to add galactosemia to the list of newborn screening tests in Arkansas (unpublished data, 1998). The data collected by the Oklahoma State Department of Health were used to develop new programs and to request an increase in funds for existing programs (unpublished data, 1998). The purpose of this letter is to encourage state health departments to continue the surveillance efforts started by the GPGSN Database Committee once this Committee is dissolved. ${ }^{2,3}$

Ing and $\mathrm{Smith}^{4}$ described the objectives of the GPGSN Database Committee as the establishment and implementation of regional standards used in data collection and the sharing of information about the delivery of services to genetic clients. The Committee's success was based, in part, on the availability of technical assistance provided to reporting centers that participated in collecting data on genetic clients. State health departments should be able to continue the efforts started by the GPGSN Database Committee because these public health institutions have the technical resources to deliver services to genetic clients and to document these efforts by using an ongoing surveillance system of data collection, analysis, and report writing. ${ }^{5}$

Four reasons support the usefulness of continuing to gather data on services provided to counseling and prenatal clients: 1 ) A regional dataset will describe more accurately the needs of genetic clients than a dataset for a single state. A multi-state surveillance system can document the extent of genetic services offered to clients who live in one state but obtain counseling or prenatal services in another state. ${ }^{1}$ 2) Analysis of large regional samples will permit states to identify small changes in the needs of genetic clients, which may precede future needs for genetic services. 3) Individual states can use these data to identify under-served populations. 4) Data sharing between states will be less expensive than the alternative of having each state increase its sample size of genetic clients.

State health departments should consider adding additional components to the surveillance of genetic services. These could include determining the effectiveness of existing programs. By evaluating the needs of genetic clients and where they reside, it will be possible to determine where these clinics should be located. This bifurcated data collection approach should result in an improved service delivery system that will be more responsive to the ever-changing needs of genetic and prenatal clients. One by-product of using data to determine program needs could be cost-containment.

Last year, the Screening and Special Services Division of Maternal and Child Health Service at OSDH formed a committee to re-design its genetic services data collection strategy. This was done to better meet the ever-changing needs of the genetic clients in Oklahoma and to determine what services have to be offered and where they should be located. The revised genetic counseling and evaluation form and prenatal form contained many items from the revised GPGSN data collection forms. Additional questions were added to evaluate other specific service needs of the genetic clients in Oklahoma. These two forms and an instructional manual were pilot tested at OSDH genetics clinics and contractor-managed patient centers for several months. While this was done, an epidemiologist designed computer databases for these two forms. A data entry technician was hired to input information from completed genetic counseling and evaluation, and prenatal genetics forms into the two computer databases. Data collection forms, manual, and database programs are available at no cost from the author. Procedures were developed to maximize data accuracy during all stages of the data collection and data entry cycle. Once every 3 months, the data from OSDH clinics and three contractor-run centers in Oklahoma City and in Tulsa are delivered to the epidemiologist at OSDH for data analysis. In the future, state data will be analyzed to determine if the services currently being offered fully meet the needs of genetic patients. If they do not, then the data will be used to determine which service delivery programs have to be modified. Another option 
will be to offer new services. Lastly, the data will be used to determine where these services, old and new, should be located. A final requirement that was placed on the re-designed surveillance system was to make sure that the counseling and prenatal datasets could be used by medical and academic researchers in the state and elsewhere. Applied research could be used to design new service delivery programs to better meet the needs of genetic clients in Oklahoma.

If other state health departments utilized the Oklahoma model, this group effort could facilitate the sharing of genetics data between states and improve the quantity and quality of services offered to counseling and prenatal clients. In one sense, state boundaries are legally assigned borders that do not directly affect the needs and conditions of genetic clients. Persons residing in one state may obtain genetic services in a nearby state for a variety of reasons. It will be in the best interest of all genetic clients, no matter where they reside, if state health departments offer services at locations that are most easily ac- cessible to them. This increased latitude would be a first step toward symbolically restoring to these genetic clients some of life's options that were denied to them at birth.

John T. Braggio, PhD

Oklahoma State Department of Health

Ohlahoma City, Oklahoma

\section{References}

1. CiPGSN Database Commulte. The Great Plans Genetics Service Network mino. mum data set report on genetics services, 1997 data. Iowa City, lowa, 1999.

2. Medney FJ, Riggle SM, Cunningham (GC, Stcrn KS, Davis JG. Prendtal genetic services: Toward a national data hase. Clin Obstet Gynecol 1943;36:510-520.

3. Neaney Fl, Chang SP. Incorporation of dinical genctic services intu the public health arena: Fducational strategies. Birth Defects 1992;28:82-89.

4. Ing PS, Smith IM. Data collection in the Great Plains Cienetics Service Network: Using limited funds to collect data from centers with varying resuurces. Proc Anmu Simp Comput Appl Med Care 1992:1:429-433.

5. Neaney FI. The future of genetics in public health. / Genet Couns 1942:1:71-80. 